\title{
Nasonia segmentation is regulated by an ancestral insect segmentation regulatory network also present in flies
}

\author{
S E Taylor and P K Dearden ${ }^{1}$ \\ Genomics Aotearoa and Department of Biochemistry, \\ University of Otago, P.O. Box 56, \\ Dunedin, Aotearoa-New Zealand \\ ${ }^{1}$ To whom correspondence should be addressed at peter.dearden@otago.ac.nz
}

\begin{abstract}
Insect segmentation is a well-studied and tractable system with which to investigate the genetic regulation of development. Though insects segment their germband using a variety of methods, modelling work implies that a single gene regulatory network can underpin the two main types of insect segmentation. This means limited genetic changes are required to explain significant differences in segmentation mode between different insects. Evidence for this idea is limited to Drosophila melanogaster, Tribolium castaneum, and the spider Parasteatoda tepidariorum, and the nature of the gene regulatory network (GRN) underlying this model has not been tested. Some insects, for example Nasonia vitripennis and Apis mellifera segment progressively, a pattern not examined in studies of this segmentation model, producing stripes at different times throughout the embryo, but not from a segment addition zone.

Here we aim to understand the GRNs patterning Nasonia using a simulation-based approach. We found that an existing model of Drosophila segmentation (Clark, 2017) can be used to recapitulate Nasonia's progressive segmentation, if provided with altered inputs in the form of expression of the timer genes $N v$-caudal and $N v$-odd paired. We also predict limited topological changes to the pair rule network. Together this implies that very limited changes to the Drosophila network are required to simulate Nasonia segmentation, despite the differences in segmentation modes, implying that Nasonia use a very similar version of an ancestral GRN also used by Drosophila.
\end{abstract}




\section{Introduction}

Embryonic segmentation has been extensively studied, both genetically and embryologically, in a number of insects and represents an excellent system with which to understand the genetic regulation of development, and its evolution (see Akam, 1989; Davis and Patel, 2002; Peel and Akam, 2003; Clark et al., 2019; Chipman, 2020 for reviews). Insect segmentation is best understood in Drosophila melanogaster, where two protein gradients, of bicoid (bcd) and caudal (cad), establish the early anterior-posterior pattern. Proteins encoded by gap genes then subdivide the body axis into broad domains, and regulate expression of the pair rule genes. Expression of the pair rule genes marks the first periodic gene expression in the embryo, these genes regulate expression of the segment polarity genes, establishing the morphological segments (Jaeger, 2011; Clark et al., 2019).

Insect trunk segmentation is broadly classified into two types: simultaneous and sequential segmentation. Simultaneously segmenting insects produce each body segment at the same time, while sequentially segmenting insects produce new segments one after the other, from a posterior segment addition zone (Davis \& Patel, 2002; Peel \& Akam, 2003; Clark et al., 2019). This simultaneous/sequential classification is an over-simplification. Nasonia vitripennis, the subject of this study, and the honeybee Apis mellifera, express segment polarity and pair rule genes in an anterior to posterior progression (Bull, 1982; Fleig \& Sander, 1988; Binner \& Sander, 1997; Osborne \& Dearden, 2005; Wilson \& Dearden, 2012; Rosenberg et al., 2014). That is, new segments are specified one after the other, but are not produced from the posterior of the embryo. This type of segmentation, progressive segmentation, is an intermediate between simultaneous and sequential segmentation. Nasonia combine progressive segmentation in the anterior with sequential segmentation in the posterior, implying that progressive and sequential segmentation can be achieved in the same species and presumably using the same genetic background (Rosenberg et al., 2014).

Modelling and empirical work implies that simultaneous and sequential segmentation can be achieved using the same molecular process. The Drosophila pair rule GRN is best understood as two networks, early and late (Clark \& Akam, 2016). The early network 
produces periodic, pair rule gene expression, while the late network converts this pair rule pattern into the segment polarity pattern (Clark, 2017). Changing the timing of network activation controls whether segmentation is simultaneous or sequential. If the timing of network activation is the same across the whole embryo, simultaneous segmentation occurs as each segment matures at the same time. If the networks are activated at different times throughout the embryo, the embryo segments sequentially (Clark, 2017; Clark \& Peel, 2018).

Transitions between these networks are thought to be controlled by the timer genes cad, Dichaete / sox21b(D) and odd-paired(opa). Cad has a well-conserved role as a posterior determinant, and regulates pair rule gene expression in many species (Copf et al., 2004; Wilson et al., 2010; Kimelman \& Martin, 2012; El-Sherif et al., 2014; Rosenberg et al., 2014; Schönauer et al., 2016; Zhu et al., 2017; Novikova et al., 2020). D is required for normal expression of some pair rule genes in Drosophila and for spider segmentation, and the expression of $D$ orthologues in the segment addition zone is conserved throughout panarthropoda (Nambu \& Nambu, 1996; Russell et al., 1996; Ma et al., 1998; Clark \& Peel, 2018; Janssen et al., 2018; Paese et al., 2018; Baudouin-Gonzalez et al., 2020). In Drosophila, opa is required for late network activation and genome-wide regulatory changes (Clark \& Akam, 2016; Koromila et al., 2019; Soluri et al., 2019). In other insects, opa is expressed in a band at the end of the segment addition zone, where a putative late network would be active, though Oncopeltus lack this pattern, implying this timer gene role may not be conserved across all insects (Green \& Akam, 2013; Xiang et al., 2017; Janssen et al., 2011; Auman \& Chipman, 2018). Together, this correlative evidence implies that the timer genes cad, D, and opa are plausibly involved in regulating segmentation across many arthropod species.

The timer gene proposal has important implications. Firstly, it provides a simple way to evolve phenotypic diversity: expressing the timer genes in different patterns causes different activation of the pair rule networks, and a switch between sequential and simultaneous segmentation (Clark, 2017; Clark \& Peel, 2018; Clark et al., 2019). Secondly, it provides a biological example of a multifunctional GRN. The multifunctionality of GRNs modelled as ordinary differential equations is well-documented. Most dramatically, the AC/DC circuit (three genes in a circuit, each repressing the one after, and one pair of genes also repress- 
ing each other) is capable of both oscillations and multistable behaviour (Panovska-Griffiths et al., 2013; Perez-Carrasco et al., 2018; Verd et al., 2019). These different behaviours are central to explaining the evolvability of the gap gene system of flies (Verd et al., 2019). Also, gene circuits with the same topology are capable of different dynamical behaviours when the weights of interactions between genes are changed (Jiménez et al., 2017). The pair rule system provides another example of a GRN which is capable of two functions: sequential and simultaneous segmentation. Both of these types of segmentation rely on the same dynamic behaviour of the GRN: the ability of the early network to oscillate within a cell. The behaviour of the GRN is unchanged, but how it is used by the organism is different (Wouters, 2003).

The lack of correspondence between a GRN topology and its dynamical behaviour means that we cannot analyse GRNs based on their structure alone (DiFrisco \& Jaeger, 2019). Instead we should dynamically model GRNs to understand them, as Clark, 2017 does for Drosophila (DiFrisco \& Jaeger, 2019). Such models are challenging to construct from the ground up for non-model species, requiring impressive amounts of experimental data which are typically lacking (Peter \& Davidson, 2016; Crombach et al., 2012; Wotton et al., 2015; Clark, 2017). Moreover, simply identifying which genes are involved in a given process, via functional data and analysis of gene expression patterns, does not give sufficient information into how these genes may interact, and how the network as whole behaves to produce patterning.

Here, we attempt an alternative approach. We use hybridization chain reaction (HCR) to describe the process of Nasonia segmentation in spatial and temporal detail (Choi et al., 2016; Choi et al., 2018). We then use an existing model of Drosophila pair rule patterning (Clark, 2017) to identify where this model can and cannot account for observed changes in Nasonia gene expression. We use this simulation-based approach to predict where changes in gene regulation are required to produce Nasonia-like patterning, and where the altered Nasonia inputs are sufficient to produce the observed changes. This computational model idealises the embryo as a one-dimensional row of cells, obeying boolean logic to determine how gene expression (protein and RNA expression and age) changes over time (Clark, 2017). 
We find that surprisingly few changes to the Drosophila pair rule GRN are required to simulate Nasonia-like patterning, implying that there may be limited topological changes to this network throughout insect evolution.

\section{Results}

\subsection{Dynamics of gene expression}

To compare Nasonia and Drosophila gene expression during segmentation, we needed a comprehensive and precisely staged description of Nasonia segmentation. To enable robust temporal and spatial characterisation of gene expression, we compared expression of each pair rule gene to the expression of $N v$-eve and $N v$-wg, alongside $N v$-sim (a marker of the ectoderm boundary, Buchta et al., 2013) for selected genes. This allowed us to stage each embryo by the number of eve and $w g$ stripes, and robustly characterise the relative timings of changes in gene expression. Stages are named by the number of wg/eve stripes: ie "eve5" embryos have five eve stripes. "Stripes" mean regions of the embryo expressing the gene of interest.

Like Rosenberg et al., 2014, we identified three distinct regions of the Nasonia embryo. In the anterior (eve stripes 1-5), Nasonia undergo progressive segmentation. Pair rule stripes of $N v$-hairy, $N v$-odd, $N v$-E75A, and $N v$-ftz are expressed in an anterior to posterior progression. The expression of $N v$-runt and $N v$-eve stripes is simultaneous: the first three stripes appear at around the same time, with $N v$-runt being expressed first (around the same time as $N v$-odd). As in Drosophila, $N v$-slp is not expressed until after other pair rule genes, at the eve4 stage. Unlike Drosophila, Nv-prd is not expressed until the eve8 stage, after frequency doubling (Keller et al., 2010). Cellularization does not commence in Nasonia until at least the eve8 stage, meaning early segmentation occurs in a pre-cellular environment (Supplemental Fig $\mathrm{S} 1)$. Frequency doubling of stripes of $N v$-slp and $N v$-eve RNA expression commences at the eve8 stage and proceeds in anterior to posterior progression, being finished in the 5th eve stripe by the wg7 stage (Rosenberg et al., 2014, Fig 1). The segment polarity genes $N v$-wg 
bioRxiv preprint doi: https://doi.org/10.1101/2021.03.23.436706; this version posted March 24, 2021. The copyright holder for this preprint

(which was not certified by peer review) is the author/funder, who has granted bioRxiv a license to display the preprint in perpetuity. It is made available under aCC-BY-NC-ND 4.0 International license.

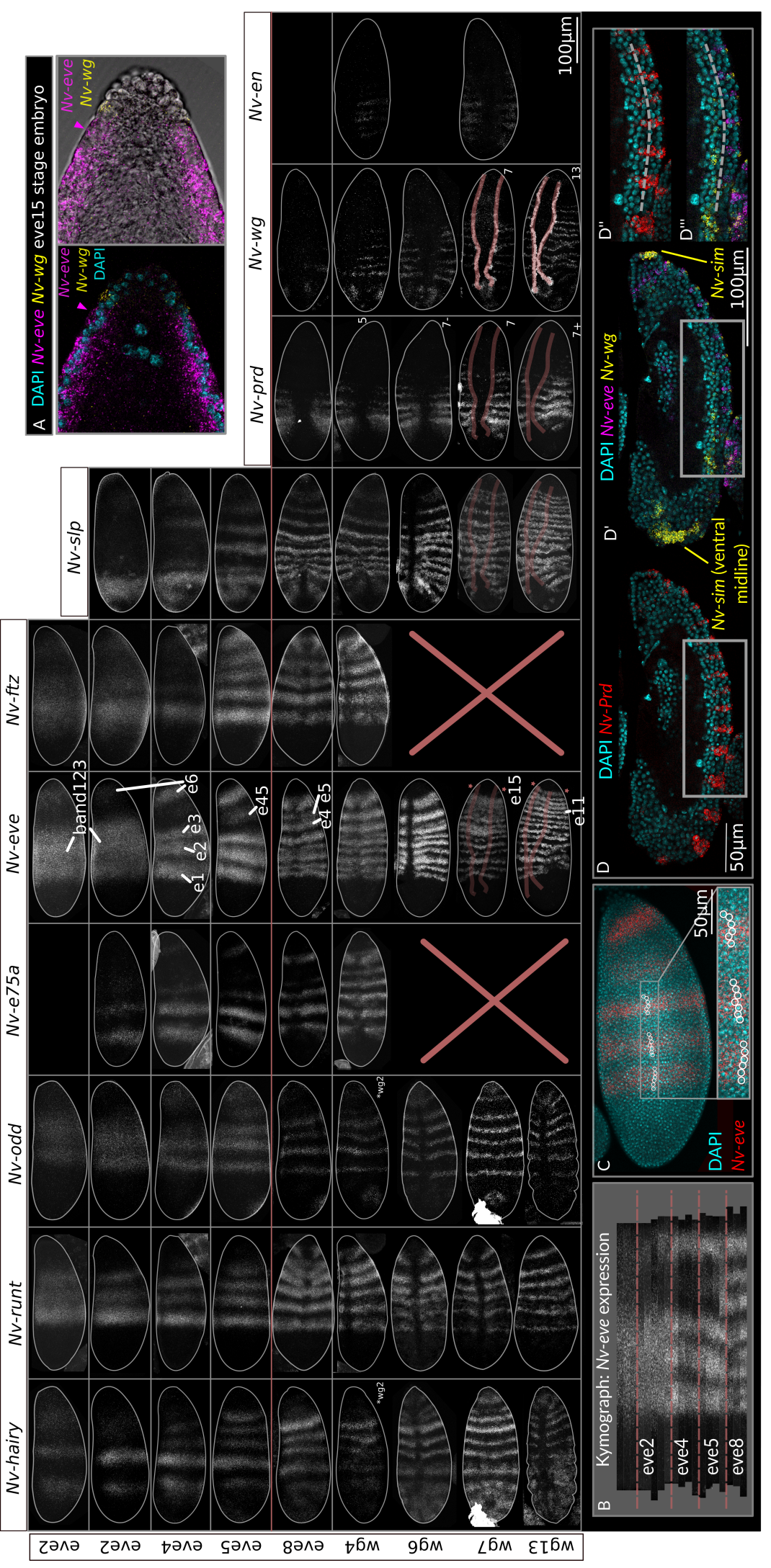

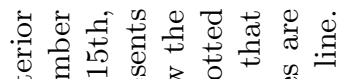

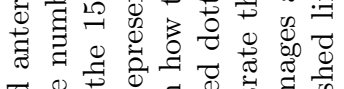

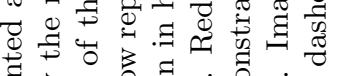

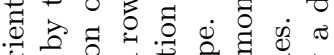

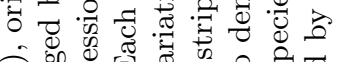

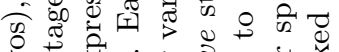

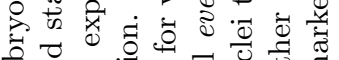
है चี

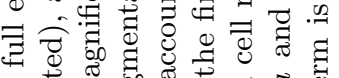

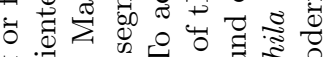

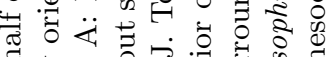
正

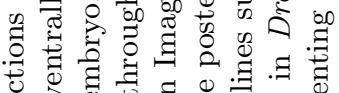

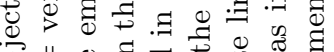

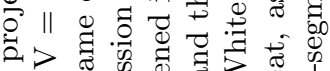

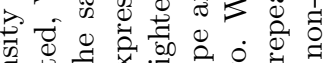

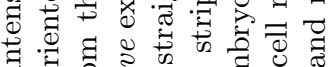

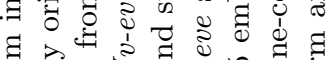

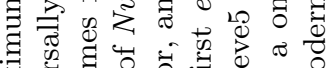
范

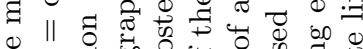

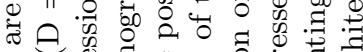

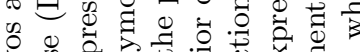

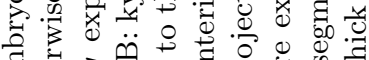
द्व

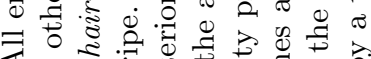

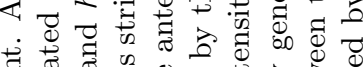

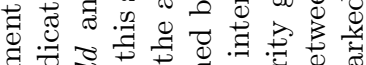

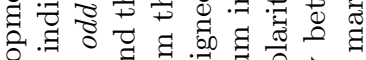
○ ช ¿

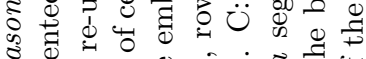

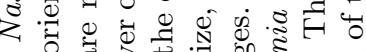

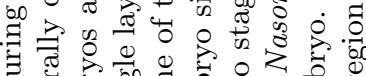

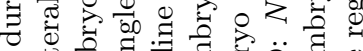

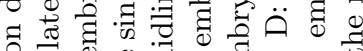
. ए

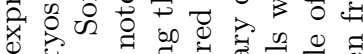
¿ है

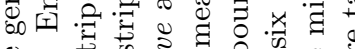

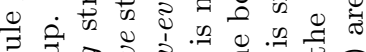
.

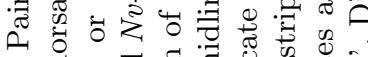

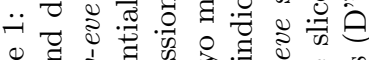

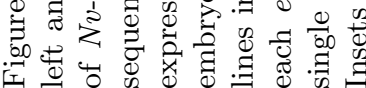


$N v$-en, and $N v$-prd likewise express RNA in stripes that form in an anterior to posterior progression, and are only expressed in the ectoderm (as demarcated by $N v$-sim expression), meaning that only the ectoderm autonomously segments in Nasonia (Fig 1). Together, these data support the notion that segmentation in the anterior proceeds in a progressive fashion, though the expression of $N v$-eve and $N v$-runt are exceptions to this.

The end of the embryo, 85-90\% egg Length (EL), behaves differently to the rest of the embryo. $N v-e^{7} 75 \mathrm{~A}$ is expressed within this region until at least the wg4 stage (we did not image $\mathrm{Nv}$-e $75 \mathrm{~A}$ stained embryos later than this), but other genes are not expressed in this stripe. The first segmental expression to be detected in this region of the embryo is that of $\mathrm{Nv}$ hairy RNA, at the wg6 stage (Fig 1). Shortly after this, the 11th $N v$-eveRNA stripe emerges from the anterior of the 6th eve RNA stripe (Rosenberg et al., 2014, Fig 1). Faint segmental expression of $N v$-slp RNA is then observed, followed by splitting of eve RNA stripe six into three stripes of single segment periodicity, separated by a stripe of $N v$-slp RNA expression (Fig S3). A sixth $N v$-odd RNA stripe is detectable at this stage, but expression is delayed and very faint (Fig S3).

The posterior regions of Nasonia embryos segment sequentially. The first expression of pair rule gene RNA in this region is in a posterior cap of $N v$-odd RNA, present from the eve4 stage. Nv-hairy RNA is expressed faintly and inconsistently the eve5 stage. Stripes of $N v$-ftz and $N v$-runt RNA appear posterior to the sixth eve stripe. Later, at the wg 4 stage, the posterior $N v$-hairy RNA expression becomes stronger and $N v$-odd RNA is excluded from the $N v$-hairy expression domain. After this, the fifteenth eve stripe appears in the posterior, appearing considerably thinner than the other stripes. This is the only sequentially appearing Nv-eve RNA stripe we observed, though Rosenberg et al., 2014 observe a sixteenth sequential stripe later. The sixteen stripes of the Nasonia are then established. This fifteenth stripe of eve RNA expression appears in the posterior of the Nasonia embryo before this region of the embryo has completed gastrulation (Fig 1A). This means that Nasonia is a long germband insect, patterning all segments before gastrulation (Davis \& Patel, 2002).

We also observed that Nasonia Nv-eve RNA stripes were 5-7 nuclei wide, in contrast to Drosophila where pair rule RNA stripes are 3-4 nuclei wide (Schroeder et al., 2011). This 
doubling in size of the primary pair rule stripes does not correspond to an increase in the final segment polarity pattern: mature $N v$-wg RNA stripes are one cell wide and separated by three nuclei (Fig 1B-D). How and why the pair rule pattern shrinks is unclear.

Though these distinct regions of the embryo differ in gene expression dynamics, there are some similarities between them. In all regions of the embryo, $N v$-hairy is the first gene we investigated to be segmentally expressed, implying that $N v$-hairy may play a key role in providing positional information to the Nasonia pair rule network. Secondly, $N v$-slp segmental expression precedes $N v$-eve stripe splitting.

\subsection{Nasonia timer gene expression can produce progressive patterning}

We wished to explain Nasonia's progressive patterning, and the expanded initial pair rule pattern. We first stained for the proposed regulators of different phases of segmentation (cad, D, and opa) (Clark \& Akam, 2016; Clark, 2017; Clark \& Peel, 2018), and used these patterns as inputs to the model of pair rule patterning.

In the first five eve RNA stripes, the expression of $N v$-cad and $N v$-opa RNA complement each other: $N v$-cad RNA retracts across the anterior-posterior axis, as $N v$-opa RNA expands (Fig 2A-F). Nv-cad RNA retracts from the broad eve stripe (band123, 30-65\%EL) region before segmental expression of $\mathrm{Nv}$-eve, and continues to retract towards the posterior as eve stripe45 splits (Fig 2A,B, S5). Nv-opa RNA is first detected in the head and stripe 1 at the eve4 stage, and expands posteriorly at the eve5 stage- shortly before $N v$-eve stripe splitting (Fig $2 \mathrm{E}$ ). $N v-D$ is expressed with different dynamics. At the eve4 stage, $N v-D$ is expressed in a broad band, from the anterior of stripe 1 to the anterior of stripe 6 . This expression persists until shortly before eve frequency doubling; at this stage $N v-D$ expression is lost in stripes 1-3 in a pair-rule-like pattern strongly resembling $D m-D$ (Nambu \& Nambu, 1996; Russell et al., 1996). $N v$ - $D$ expression is retained around stripe4/5 until these stripes start to undergo frequency doubling. Curiously, $N v$-cad and $N v$-D are never expressed within stripe 6 (which has unusual eve dynamics); this stripe progresses straight to $N v$-opa expression (Fig $2 \mathrm{E}-\mathrm{G})$. 
bioRxiv preprint doi: https://doi.org/10.1101/2021.03.23.436706; this version posted March 24, 2021. The copyright holder for this preprint (which was not certified by peer review) is the author/funder, who has granted bioRxiv a license to display the preprint in perpetuity. It is made available under aCC-BY-NC-ND 4.0 International license.
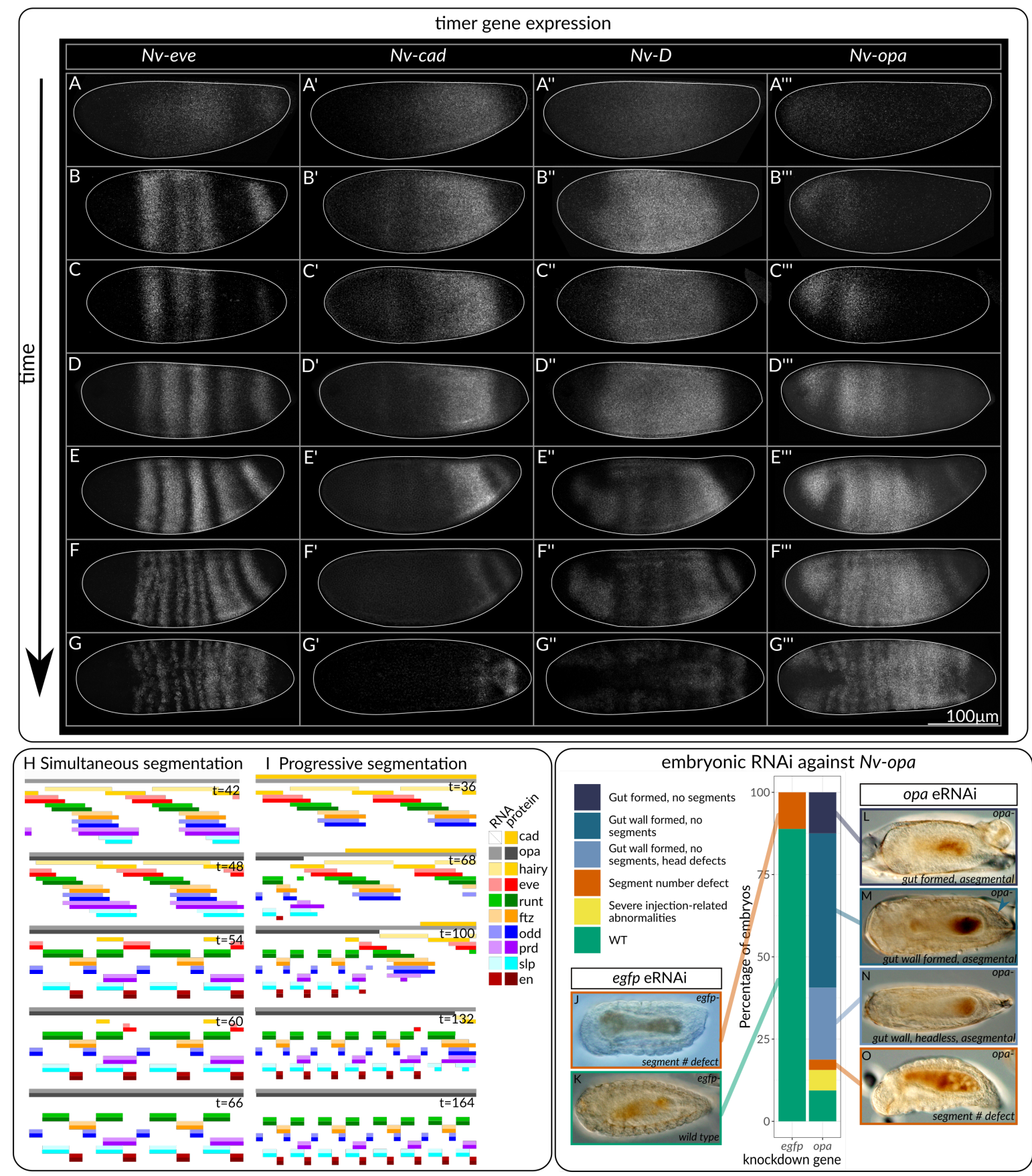

Figure 2: Timer gene expression in Nasonia recapitulates progressive patterning when modelled. A-G: Maximum intensity projections of embryos stained with the timer genes $N v$-cad, $N v$ - $D, N v$-opa, staged to $N v$-eve. All embryos are laterally oriented, anterior left, except $\mathrm{G}$ which is ventrally oriented. $\mathrm{H}$ and I: simulation of broad pair rule stripes combined with simultaneous (H) and sequential/progressive (I) timer gene dynamics. J-O: $N v$-opa is required for morphological segmentation. All embryos are oriented anterior left, ventral view. Arrow in $\mathrm{M}$ indicates the position of the gonads. 
These opa and cad expression dynamics were able to recapitulate Nasonia-like progressive patterning, if simulated in a model containing Nasonia's broad (6-cell wide) pair rule stripes (Fig 2H, I). In this model, stripes mature in an anterior to posterior progression, characteristic of progressive segmentation. The cad and opa dynamics are crucial to this progressive patterning: the same network simulated with broad pair rule stripes, but activated in a simultaneous manner results in an embryo that segments simultaneously, but has a final pattern doubled in size from the Drosophila model, ie a 16-cell repeat of gene expression (Fig 2H). The progressive model exhibits the Drosophila and Nasonia-like eight cell repeat. Note that the phasing of the late network in this simulation is Drosophila, not Nasonia, like.

To further investigate the role of the timer genes in Nasonia segmentation, we performed embryonic RNAi (eRNAi) against $N v$-opa. We identified two phenotypes following opa eRNAi: a total lack of segments within the embryo, and apparent defects in head formation. To distinguish between developmental arrest prior to morphological segmentation, and segmentation defects, we used DIC imaging to identify two morphological markers that appear after segmentation in wild type embryos: presence of the gut wall, and (where visible) gonads (Bull, 1982). Surviving embryos (8/67 egfp-, 32/64 opa-) were scored into six classes. Some embryos had an obvious gut, no gut wall, and no segments. These embryos were only present after injection with opa dsRNA, and could represent either developmental arrest at a stage prior to morphological segmentation, or a segmentation defect. Many embryos successfully formed the gut wall, which forms after morphological segmentation (Bull, 1982), but lacked any distinguishable morphological segments. Some of these embryos also lacked a head. A small number of egfp and opa injected embryos had defects in the number of segments. Control embryos with these phenotypes also exhibited cytoplasmic leakage, suggesting that this phenotype was caused by the injection procedure itself. Completely asegmental embryos occurred in $69 \%$ of surviving opa- embryos and never occurred in egfp-embryos. These data show that $N v$-opa is required for morphological segmentation in Nasonia, supporting its proposed role as a key regulator of segmentation. The defects in head formation are consistent with the expression of $\mathrm{Nv}$-opa in the head (Fig $2 \mathrm{~A}-\mathrm{G}$ ), and its requirement for head formation in Tribolium (Clark \& Peel, 2018). 


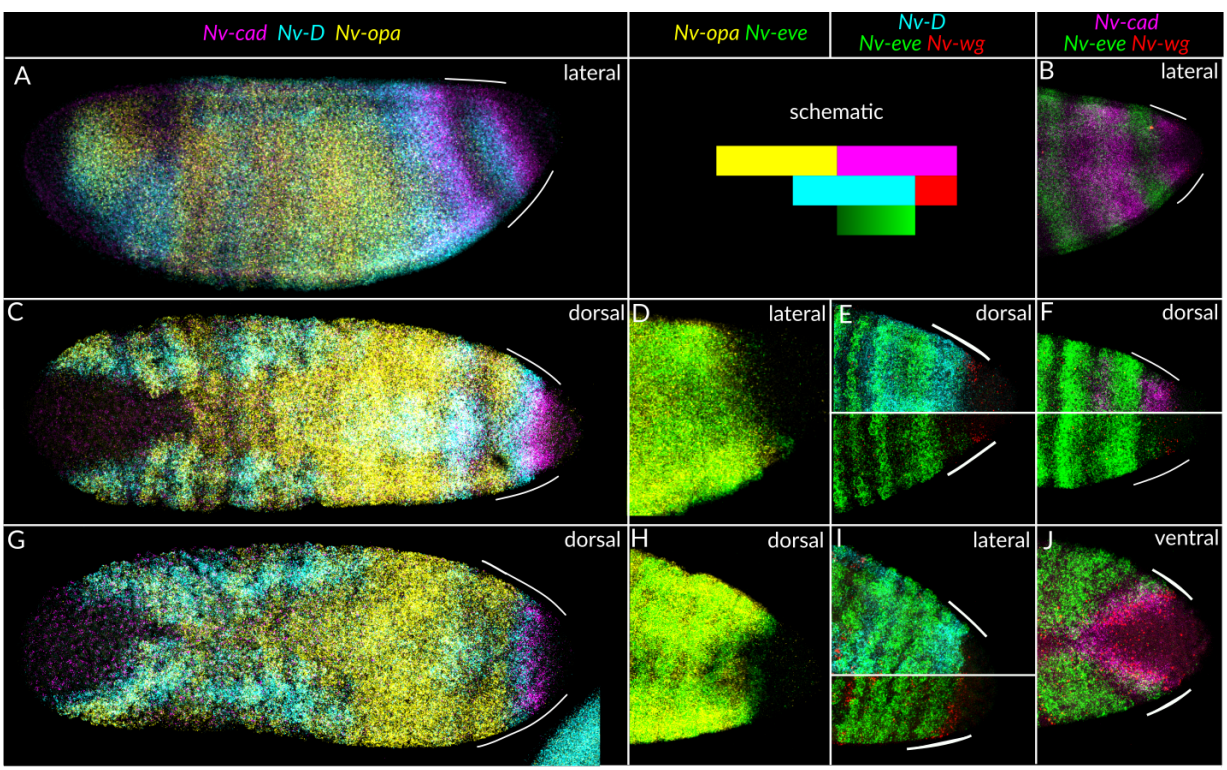

Figure 3: Timer gene expression in the posterior of the embryo is similar to other sequentially segmenting species. All embryos are partial or full maximum intensity projections, anterior left. Left panels (A, C, G): whole embryos. Right panels: cropped to just show the posterior.

We then attempted to understand how Nasonia undergo sequential segmentation / the appearance of stripe 15, again by staining for timer gene expression. Before stripe 15 appearance, at the eve9 stage, $N v$-cad and $N v$-D RNA are expressed in overlapping stripes behind the eve6 stripe, with $N v$-cad most posterior and overlapping with $N v$-wg RNA (Fig 3C). Later, at the eve9 stage, the fifteenth eve stripe appears within the $N v$-cad/Nv-D domain, anterior to the posterior $N v$-wg stripe (Fig 3C-F). At this stage, a posterior $N v$-opa stripe is visible within the 6th eve stripe. This $N v$-opa expression is stronger at the ventral ends of the embryo, and is expressed up to the anterior end of the fifteenth eve stripe (Fig 3D). At the eve15 stage, the eve stripe is still expressed within the $N v$ - $D$ and $N v$-cad domain, but $N v$-opa has expanded posteriorly to be co-expressed with eve at the anterior of the eve stripe. Thus, Nasonia possess a similar spatial sequence of the timer genes as Tribolium: a spatial sequence of gene expression in the order $N v$-wg $N v$-cad $N v$-D Nv-opa. This pattern was not simulated, because it is qualitatively similar to the sequential model of segmentation (Clark, 2017; Clark \& Peel, 2018).

With the exception of stripe 6, then, the Drosophila GRN combined with the Nasonia cad and opa expression patterns are able to recapitulate the Nasonia dynamics- both the 
existence of progressive segmentation in the anterior, and sequential segmentation in the posterior. However, this analysis relies on two critical assumptions. Firstly, it relies on there being two different pair rule GRNs in Nasonia, with qualitatively different behavioursoscillatory vs non-oscillatory expression of genes. Secondly, it assumes that the pair rule networks of Nasonia and Drosophila are reasonably similar. In the following sections, we use our description of Nasonia segmentation to address both these points.

\subsection{Organisation of Nasonia pair rule gene expression}

Assessing whether two GRNs act in a given process is challenging. We observe, however, startling coordination between structural and behavioural changes in gene expression, which we interpret as meaning that two GRNs are acting in Nasonia expression. For instance, frequency doubling (of $N v$-slp and $N v$-eve) and expression of segment polarity genes begins at the eve8 stage, and occurs in an anterior to posterior progression within the embryo (Fig 1). Segmental expression of $N v$-prd also begins at this stage, and again occurs in an anterior to posterior progression, with segmental expression of $N v$-prd being detectable at the same time as $N v$-eve and $N v$-slp frequency doubling (Fig 1). There is also a dramatic shift in the relative expression of $N v$-slp and $N v$-eve: these genes go from being co-expressed to being strongly anti-correlated within the embryo, implying that the regulatory relationship between these genes has changed (Fig 5). These changes are tightly coordinated throughout the embryo, implying that they share a common cause. Expression of $N v$-opa precedes these changes, implying that $N v$-opa may cause these changes (Fig 2), an observation strengthened by the fact that $N v$-opa is required for morphological segmentation. We also observe a change in dynamics at the eve8 stage. The second and third pair rule stripes shift forwards until the eve8 or eve9 stage, then stop (Fig 1B, Fig 4). This change in gene expression dynamics implies that the behaviour of the networks underpinning these gene expression patterns has changed. Together, these data imply that Nasonia possess two pair rule GRNs (or functional/pragmatic modules of a larger pair rule GRN (Verd et al., 2019)) with two distinct behaviours. 


\subsection{Conservation of the early segmentation network}

We then investigated the conservation of these two pair rule networks. The primary pair rule genes involved in the early network, $N v$-eve, $N v$-hairy, $N v$-odd, $N v$-runt, and $N v$-ftz, are expressed in very similar patterns relative to each other as in Drosophila (Fig 4A-D, Rosenberg et al., 2014). Nasonia pair rule RNA stripes still overlap and are expressed in the order $N v$-hairy $\rightarrow N v$-eve $\rightarrow N v$-odd/Nv-ftz $\rightarrow N v$-runt $\rightarrow N v$-hairy (see Fig 4A-D). To concisely display pattern maturation over time, we quantified gene expression along the midline of the embryo in Image $\mathrm{J}$ (averaging across $20-50 \mu \mathrm{m}$ ), and plotted these intensities as colour gradients. This analysis revealed that the hairy-eve-runt-odd pattern develops over time, from being present only in the very anterior at the eve2 stage, to being present in the fourth and fifth stripes at the eve9 stage (Fig 4E-I). The expression of $N v$-slp and $N v$-prd RNA, as mentioned above, has changed compared to Drosophila. Nv-slp RNA is expressed within, not between, $N v$-eve RNA stripes (Fig $4 \mathrm{C}$ ), and $N v$-Prd RNA is not expressed until later in segmentation (Fig 1). In addition, we identified early segmental expression of $N v$ E75a RNA anterior to eve RNA (Fig 4D), implying that this gene may also be involved in early segmentation.

These changes in $N v$-slp and $N v$-prd expression would not impact the behaviour of the early network, as these genes do not cross-regulate other pair rule genes (Clark, 2017). Expression of extra gene/s, eg $N v-e 75 a$, is addressed below. Thus, the Nasonia expression of these genes is consistent with a topology very similar or identical to the Drosophila ones, and does not imply a change in behaviour of the Nasonia network.

Despite the above, we know that the Nasonia network is capable of different behaviour to that of Drosophila: sequential patterning. This would rely on oscillatory expression of the primary pair rule genes. We therefore looked for evidence that Nasonia have oscillatory pair rule gene expression, where oscillatory gene expression is expression of the full hairyeve-runt-odd sequence within a single cell. We found no oscillatory expression in the anterior or posterior of Nasonia. In the posterior, sequential expression begins with a cap of $N v$-odd expression (Rosenberg et al., 2014, Fig 1). At the eve8 stage, a posterior stripe of $N v$-runt 
bioRxiv preprint doi: https://doi.org/10.1101/2021.03.23.436706; this version posted March 24, 2021. The copyright holder for this preprint (which was not certified by peer review) is the author/funder, who has granted bioRxiv a license to display the preprint in perpetuity. It is made available under aCC-BY-NC-ND 4.0 International license.
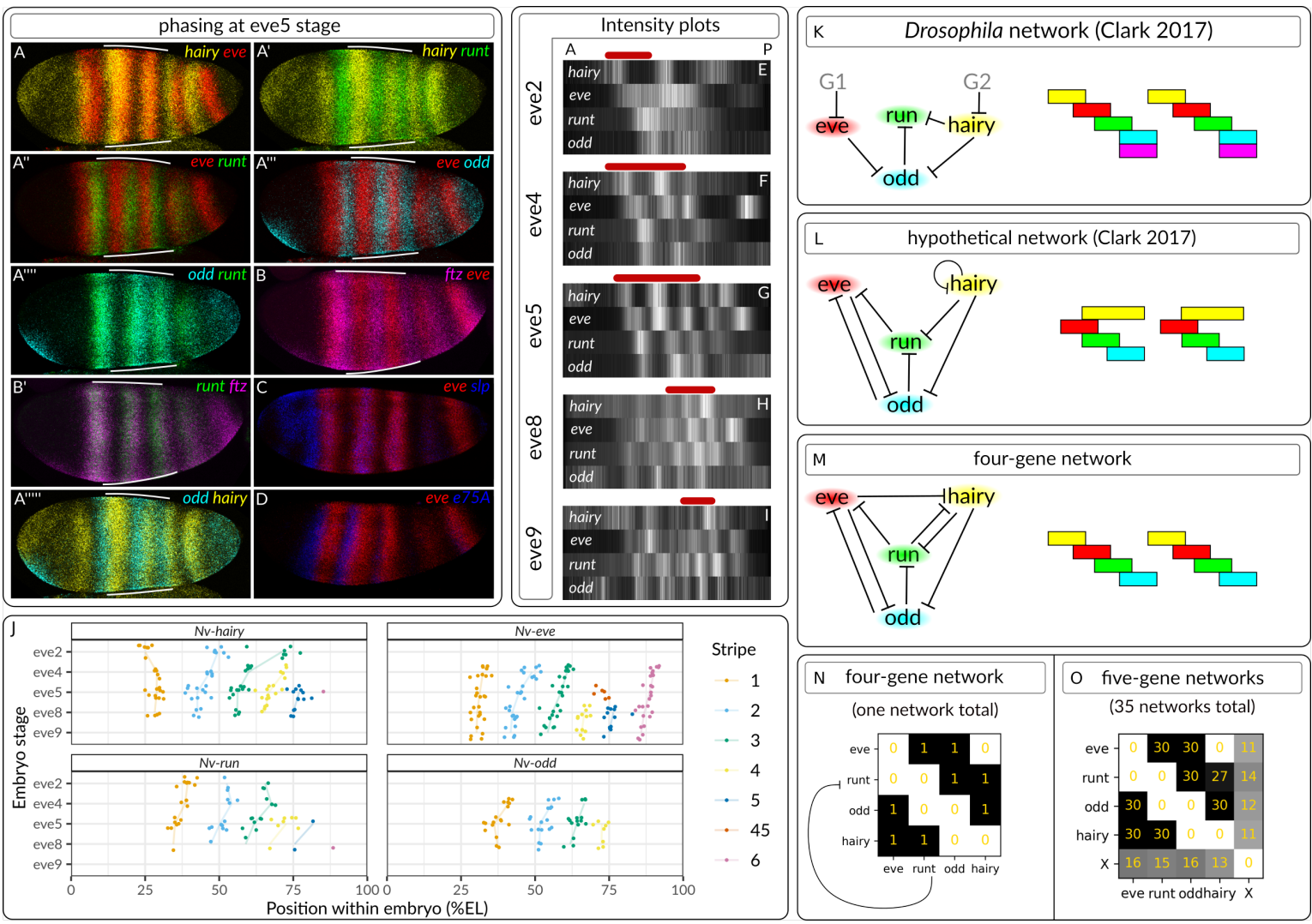

Figure 4: Phasing of the Nasonia early network closely resembles that of Drosophila. A-D: maximum intensity projections of embryos stained for pair rule genes. All embryos are laterally oriented, anterior left. White bars indicate the stripes which have the pair rule pattern established. E-I: Intensity plots describing gene expression at different embryo stages. Signal is averaged from $10-50 \mu \mathrm{m}$ along a line following the curvature of the embryo, and are normalised 0 and 1. Background is defined as the signal intensity present in the head (where no genes except for hairy are expressed) and is set to zero. White bars indicate high intensity of gene expression. A: anterior. P: posterior. Red bars indicate the region of the embryo with the pair rule pattern established. Note that eve8 and eve9 embryos are beginning to undergo gastrulation so anterior stripes are more disordered. J: Forward movement of pair rule stripes within the embryo over time. Central positions of stripes were gauged by eye from straightened intensity profiles along the middle of the embryo. Black bars indicate standard error of the mean, coloured lines join the mean at different stages. K-M: network topology (left) and associated stable gene phasing (right). N: Topology of the four-gene network visualised as a matrix. Repressive interactions are read $\mathrm{X}$ to $\mathrm{Y}$, that is, run represses eve. O: Five-gene networks possessing a given genetic interaction. Frequency of interaction is given in gold. Gene X: unconstrained fifth gene initialised with the same expression as hairy. 
forms anterior to this $N v$-odd cap. A stripe of $N v$-hairy then subdivides the $N v$-odd cap, and the fifteenth eve stripe is expressed. This produces a spatial pair rule gene sequence (anterior to posterior, $N v$-runt, $N v$-odd, $N v$-hairy, $N v$-eve, as well as a temporal $N v$-odd-Nv-hairy- $N v$ eve sequence in the region of the fifteenth eve stripe, again consistent with the sequence from Drosophila. However, no cell goes though the full gene sequence.

In the anterior, we were unable to determine gene expression in each cell over time, because of the shifting domains of gene expression and the lack of cell markers. We instead quantified the changes in stripe position over time, reasoning that changes in gene expression within a single cell would also be visible as changes in the position of stripes of pair rule gene expression within the embryo. Cells in the Nasonia anterior also do not go through a full cycle of gene expression, because though pair rule stripes shift forwards in the embryo, no pair rule genes move forwards by an entire segment. That is, no position within the embryo completes a full cycle of pair rule gene expression, starting and ending at the same gene. If this behaviour was due to stability, we would expect gene expression to arrest at one pair rule gene. It does not: all four pair rule genes where we had sufficient data to assess stripe shifts exhibit forward shifts in gene expression (Fig 4J). Thus, the Nasonia early network appears to be capable of oscillatory gene expression, though whether this is driven by the anterior movement of gap gene expression in Nasonia, or the intrinsic dynamics of the network, is unclear (Pultz et al., 2005; Brent et al., 2007; Clark, 2017; Ray et al., 2019). It is also worth pointing out that oscillations that do not go through a full cycle of gene expression (damped oscillations) can pattern segments in the gap gene system of Drosophila (Verd et al., 2018).

The early network topologies of Clark, 2017 are either incapable of sequential segmentation (the Drosophila network, Fig 4K), or inconsistent with the observed gene expression patterns in Nasonia (the hypothetical network, Fig 4L). To identify a four-gene topology capable of sequential segmentation and the Nasonia phasings, we performed a computational screen. Topologies were classed as potentially sequential if, after 100 time-points of the simulation (about 2.5 full oscillations), they went through the sequence hairy $\rightarrow$ hairy/eve $\rightarrow$ eve $\rightarrow$ eve/odd $\rightarrow$ odd $\rightarrow$ odd/runt $\rightarrow$ runt $\rightarrow$ runt/hairy $\rightarrow$ hairy. This screen identified one topology capable of producing sequential segmentation from normal inputs- the topology in 
Fig 4M. This shows that the gene phasing observed in Nasonia is consistent with at least one GRN topology capable of sequential segmentation. Unlike the hypothetical network, this potential network requires the positions of every pair rule gene to be provided to the simulation in order to produce normal patterning (Figure S4), meaning that simultaneous patterning using this topology requires an extensive (presumably gap gene-mediated) spatial pre-pattern. This topology also does not resolve the potential use of $N v$-e $75 a$ and other novel pair rule genes, which could change the behaviour of the network. Accordingly, we screened for five-gene topologies capable of sequential segmentation, with the fifth gene (gene X) initially co-expressed with hairy but with no final restrictions on its expression. After filtering out networks with topologies identical to the four-gene network, this analysis identified 35 potential networks, for which the frequency of genetic interactions are presented in Fig $4 \mathrm{O}$. Although some genetic interactions remained impossible for this model formulation, the addition of the extra gene provided flexibility to the network, ensuring that no genetic interaction was present in every predicted network. Though this analysis is unlikely to have identified every possible five-gene network capable of sequential segmentation, as the initial conditions of gene X could be very different to our assumptions, it does demonstrate that there are various possible networks incorporating different genes (eg $N v$-e $75 A$ ). We draw two conclusions from the above analysis. Firstly, we identified one potential network capable of producing both the Nasonia gene expression patterns and sequential patterning. Secondly, addition of one extra gene (plausibly $N v$-e $75 a$ ) provides more flexibility to genetic interactions in the early network but does not have to change the behaviour of the network.

Taken together, this analysis shows that the Nasonia network could be capable of oscillatory gene expression, and sequential segmentation.

\subsection{Topological changes required to explain some aspects of Nasonia late network}

Unlike the early network, there are changes to gene expression in the Nasonia late network. Different genes undergo frequency doubling in Nasonia compared to Drosophila. In Nasonia, only $N v$-eve, $N v$-slp, and $N v$-runt undergo frequency doubling, whereas in Drosophila, Dm-odd 
also doubles (Schroeder et al., 2011; Rosenberg et al., 2014). Nv-odd is expressed as a twocell wide stripe, not one-cell wide, while secondary $N v$-runt stripes are expressed very briefly and are only one cell wide (Fig 1, Rosenberg et al., 2014). In addition, $N v$-slp and $N v$-odd overlap (Fig 5E). We wished to know whether the observed changes in expression in the late network could be explained by changes in input to the late network- ie the altered positioning of $N v$-slp, $N v$-prd, and $N v$-E75A or whether they require changes in gene regulation.

We approached this problem via simulation. We used an $\mathrm{R}$ package, BoolNet (Müssel et al., 2010), to model the output of the Drosophila network under various input conditions. This package ignores the gap between RNA transcription and protein translation, unlike Clark, 2017's model. We used this abstraction to avoid arbitrarily assigning protein and RNA ages when initialising models. Modelling Drosophila gene expression at the $\mathrm{t}=36$ timepoint in this way produces gene expression patterns identical to the existing model if $s l p$ expression in cell 1 is ignored (this expression would decay naturally in the next few timepoints) (Fig 5A). Modelling the Nasonia eve5 gene expression was able to produce some aspects of Nasonialike patterning of the even-numbered segment. In this segment, the main difference between Drosophila and Nasonia is that odd stripes are two cells wide, and that these cells are co-expressed with slp (Fig 5D). Additionally, towards the end of segmentation, Nv-runt is expressed in one-cell wide stripes, co-expressed with $N v$-wg. The altered inputs to the evennumbered parasegments are able to recapitulate these patterns: the lack of $s l p$ expression posterior to odd means that the odd and ftz domains are not repressed posteriorly, and these genes remain as two/three cell stripes. In these simulated cells, slp and prd expression do not resolve properly- $s l p$ because, in Nasonia, slp and odd are co-expressed, a pattern inconsistent with the mutual repression of these genes in Drosophila and the Drosophila model used. Prd never becomes expressed because prd expression is only possible in cells already expressing prd and we supply no $N v$-prd expression to the model. As in the Drosophila model, secondary eve stripes do not appear. In summary, this analysis shows that the Nasonia inputs to the Drosophila network are able to produce some of the altered Nasonia gene expression.

We were unable to produce proper patterning in odd-numbered segments. Second runt and odd stripes form, which are not present in Nasonia embryos, and odd is mis-positioned. 
bioRxiv preprint doi: https://doi.org/10.1101/2021.03.23.436706; this version posted March 24, 2021. The copyright holder for this preprint (which was not certified by peer review) is the author/funder, who has granted bioRxiv a license to display the preprint in perpetuity. It is made available under aCC-BY-NC-ND 4.0 International license.
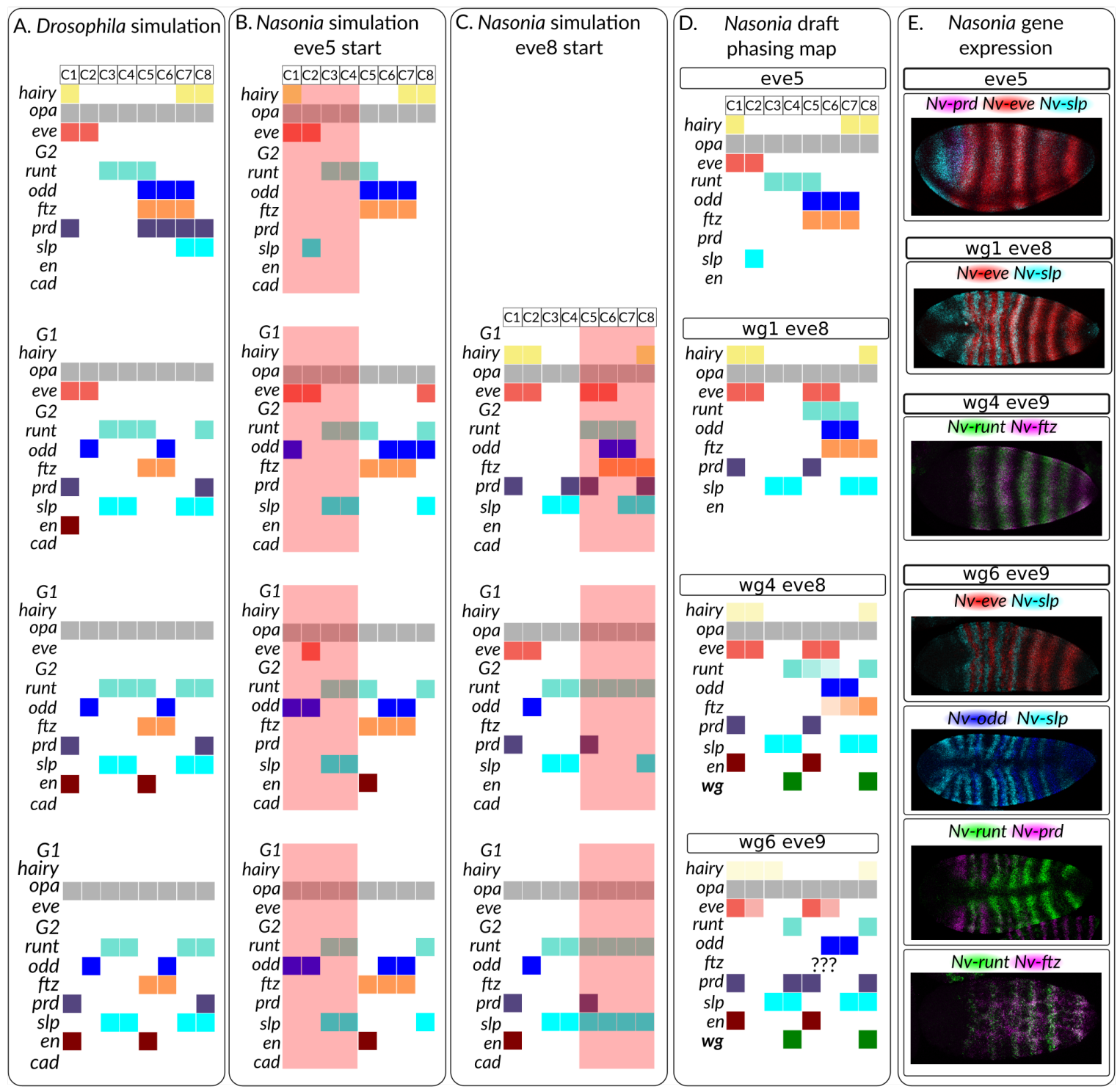

Figure 5: Altered inputs can recapitulate some aspects of late Nasonia segmentation. Note that each cell takes different numbers of time-steps to reach final output states so the temporal information depicted is not necessarily accurate. A: Drosophila simulations based on gene expression at the $t=36$ timepoint of simulations in Clark, 2017. Note that in cell 1 (C1), slp expression was omitted to ensure proper segmentation and because expression in this cell would decay without regulatory input. B: Nasonia simulations based on expression at the eve5 stage. Red bar indicates region where proper patterning is not produced, likely because of the lack of prd in the input. C: Nasonia simulations, starting with gene expression at the eve8 stage. Red bar indicates region where proper patterning is not produced. D: Draft map of gene expression in the Nasonia embryo. Map is based primarily of gene expression in the first four segments, which is representative of expression throughout the embryo. The map is produced based on comparisons to $N v$-eve and $N v$ - $w g$, so other gene-gene comparisons may not be strictly accurate. E: selected HCRs showing relative expression of genes. All embryos are maximum intensity projections, anterior left. See Figs S6, S7, S8 for the rest of the dataset. 
To check that this was not due to the lack of Prd, we initialized the model with Nasonia inputs from the eve8 stage. This produced Drosophila, not Nasonia, gene expression patterns, and incorrect phasings in C4-C8 (compare Fig 5A-C). This means that the Nasonia inputs, at both the eve5 and eve 8 stage, cannot produce the Nasonia outputs, and therefore that the Drosophila model cannot produce the Nasonia odd-numbered stripe gene expression. Involvement of an additional gene is required to produce these changes. In the Nasonia cell expressing odd, eve is the only gene to be expressed, both in simulations and the embryo (Fig 5B-D). Eve is thus the only modelled pair rule gene that could turn odd off in this cell. However, we know that a repressive relationship between odd and eve cannot exist in Nasonia, because these genes are stably co-expressed (Fig 5D). Thus, additional genes must be involved to repress odd in this cell. A similar argument holds for runt: in cells 3-4, only slp is co-expressed with runt so only slp could repress runt. Again, there cannot be a repressive interaction between these genes, because $s l p$ is co-expressed with runt in cell 8 .

This means that the Drosophila network can produce Nasonia's expanded odd and ftz expression, as well as the smaller runt domains. However it cannot recapitulate the lack of odd doubling, and general patterning in cells 1-4. Changes in gene regulation possibly related to e75a and/or other genes are required to explain these features.

\section{Discussion}

Here, we explore the conservation of the pair rule GRN in Nasonia and Drosophila. Despite morphological and gene expression differences between Nasonia and Drosophila segmentation, we found that altered inputs to a largely conserved Drosophila network can explain these differences. The general organization of the pair rule genes into two networks is likely conserved in Nasonia, providing the first evidence of such organization outside Drosophila. In addition, though there are changes to the genes expressed in the early network, the early network still appears to behave in a similar way to Drosophila. We were able to recapitulate the Nasonia segmentation dynamics- progressive patterning- using the Nasonia timer gene expression patterns. Finally, changes in the input to the late network are able to recapitulate 
some, but not all, changes in Nasonia segmentation; there must be some topological changes to the late network.

\subsection{Timer gene hypothesis explains the dynamics of Nasonia segmenta- tion}

Clark, 2017 had previously shown that a slightly modified Drosophila pair rule GRN can perform both simultaneous and sequential patterning. That is, either spatial or temporal information can be used to produce patterning. In simultaneous patterning, the information is spatial, in the form of gap gene mediated patterning of pair rule genes. In sequentially segmenting insects, a temporal pattern is used (Clark, 2017; Clark \& Peel, 2018; Clark et al., 2019). Our work reinforces and extends both these points. Firstly, we analyse an insect which combines multiple modes of segmentation (Rosenberg et al., 2014). In the anterior, Nasonia undergo progressive patterning, while in the posterior, they undergo sequential segmentation. Anterior expression of timer genes was used to model progressive patterning, while in the posterior, the timer genes are expressed in a similar spatial sequence to the other sequentially segmenting insect studied, Tribolium (Clark \& Peel, 2018). Presumably, the GRN used does not change between the anterior and posterior of Nasonia. Together, these findings mean that the expression of timer genes can explain the unique segmentation dynamics of Nasonia.

Secondly, inspired by Nasonia patterning, we provide a way to combine a spatial and temporal pre-pattern, producing what we have called progressive patterning. Broad pair rule stripes (an expanded spatial pattern) correct for the temporal pattern, an expansion/retraction of $c a d$ and opa expression, ultimately leading to progressive patterning.

This finding has important implications for the evolution of segmentation. It means that full simultaneous and sequential segmentation are likely two ends of a spectrum, rather than distinct types of segmentation. Such a spectrum is implied in the findings of Clark, 2017; Clark and Peel, 2018: if simultaneous and sequential segmentation share a mechanistic basis or GRN, then intermediates could exist. Small changes to the expression of the timer genes, and the width of pair rule stripes, can have dramatic impacts on how the pair rule GRN 

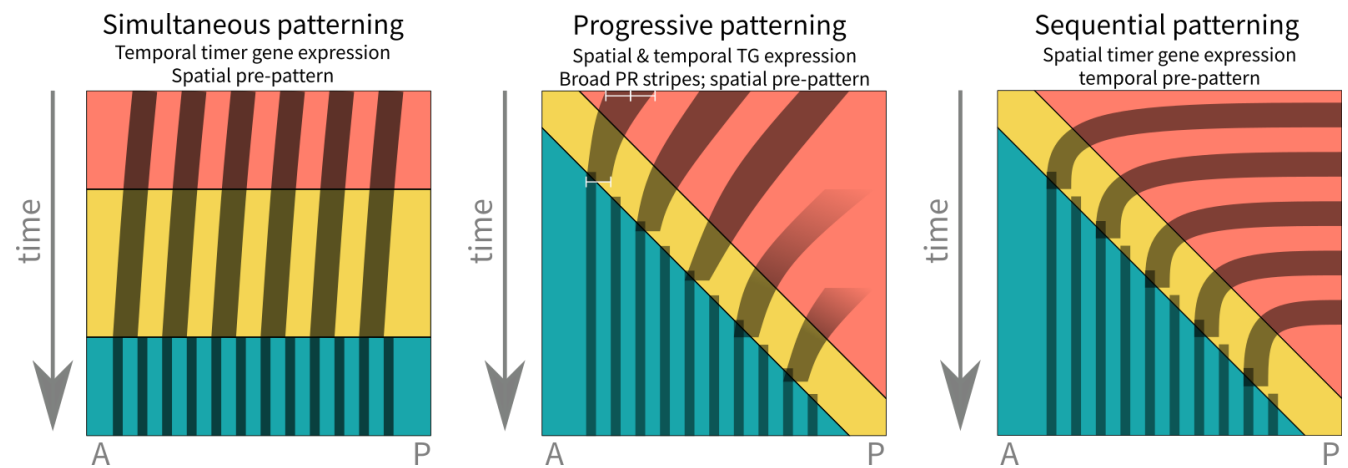

Figure 6: Schematic of simultaneous, sequential, and progressive segmentation. Adapted from Clark et al., 2019

behaves, and so to the dynamics of segmentation, providing a simple and elegant method of evolving phenotypic variation.

\subsection{Topological changes to network}

Unsurprisingly, modelling the dynamics of Nasonia segmentation was unable to recapitulate differences in gene phasings between Nasonia and Drosophila. Our data, combined with modelling, provide insight into potential regulatory changes achieving these altered expression patterns in Nasonia. We found that the general organisation of the Drosophila pair rule network was conserved in Nasonia, as apparent changes to gene regulation are coordinated within the embryo, and correlate with $N v$-opa expression. This conservation of GRN structure implies that such a structure is evolutionarily important (Clark \& Akam, 2016; Clark \& Peel, 2018). It also provides support for the timer gene idea, which relies on two GRNs with different behaviours being activated in different ways, to achieve patterning (Clark, 2017).

We found no evidence for topological changes to the early network, aside from the changes in $N v$-slp and $N v$-prd expression. The primary pair rule genes are expressed in the same order as Drosophila, and like Drosophila, shift anteriorly within the embryo over time (Fig 4A-J, (Clark, 2017; Lim et al., 2018)), though whether this is driven by gap genes is unclear (Pultz et al., 2005; Brent et al., 2007; Ray et al., 2019). This means that the Nasonia early network can be modelled by the Drosophila GRN. We identified a four-gene network topology capable of sequential segmentation, meaning that the expression patterns observed are consistent 
with sequential segmentation. Finally, we found that addition of extra genes to this network does not change the behaviour of the network, meaning the possible addition of extra genes, eg. Nv-e $75 a$, to the early network does not imply a change in network behaviour. This shows that the Nasonia early network is similar in behaviour to the Drosophila one, and that such behaviour is consistent with a network capable of sequential segmentation.

We also found that the Drosophila late network can explain some, but not all, aspects of Nasonia segmentation. The expanded odd and ftz domains in Nasonia can be explained by the Drosophila network and Nasonia's altered inputs to it, while the lack of odd frequency doubling requires topological changes to the network. We are able to predict some of these changes. Firstly, Nasonia must lack strong mutual repression between $s l p$ and odd to maintain stable co-expression of these genes. Secondly, there must be another gene or genes patterning the anterior parasegment/cells 1-4, to prevent frequency doubling of $N v$-runt and $N v$-odd. Thirdly, the regulation of $N v$-eve must change to allow eve frequency doubling. Crucially, there is no evidence that such changes would change the behaviour of the network- the Nasonia late network still has a similar end point: stable expression of segment polarity genes (Figs 1, S2). This is consistent with previous work, as empirical and modelling work imply that the segment polarity network is stable and well conserved (Von Dassow \& Meir, 2004; Choe \& Brown, 2009; Janssen \& Budd, 2013; Green \& Akam, 2013; Vellutini \& Hejnol, 2016; Auman \& Chipman, 2018; Clark et al., 2019).

\subsection{Studying GRNs}

These findings reinforce a number of important points. These behaviours of the Drosophila and Nasonia pair rule networks can only be understood using dynamical modelling ((Clark, 2017), Figs 4, 2), reinforcing the time-dependent nature of the genetic regulation of development, and the necessity of using modelling as a tool to understand GRNs (DiFrisco \& Jaeger, 2019; Briscoe, 2019). Secondly, the late GRN is input-dependent: changes to the inputs of this GRN are able to explain some (but not all) changes in the Nasonia gene phasings of the late network. Again, this reinforces the need to use modelling alongside empirical research, and in particular the power of using modelling to understand developmental processes in 
less-studied species such as Nasonia.

We also found that though the dynamics of Nasonia patterning can be recapitulated using the Drosophila network, the relative patterns of gene expression cannot be fully accounted for by such an analysis.

Are the Nasonia and Drosophila GRNs homologous? They share common descent: the involvement of pair rule genes in arthropod segmentation is extensively documented, and is at least as old as holometabolous insects (Dearden et al., 2006). It is unclear whether these GRNs are structurally homologous, as we collected no data on the interactions between Nasonia pair rule genes. Moreover, the structure of a GRN provides little information as to the behaviour of such a GRN within a cell, and what traits it can allow in an organism (DiFrisco \& Jaeger, 2019). However, we do provide evidence that the two GRNs are functionally homologous, that is, they behave the same way within the organism. The early network, as argued above, looks very similar to Drosophila. The late network is more difficult: there appear to be some changes to gene regulation. However, the output of segmentation is the same (expression of the segment polarity genes), and there is no evidence for a change in the dynamics of stripe expression (eg anterior movement of stripes). We think that this conservation of output and behaviour means that the function of the late network is unchanged. Thus, the Drosophila and Nasonia GRNs may be thought of as functionally homologous (DiFrisco \& Jaeger, 2021).

\subsection{Conclusion}

Overall, we find that remarkably few changes to the Drosophila pair rule GRN are required to simulate Nasonia patterning. Nasonia's progressive patterning can be recapitulated by changing the $N v$-cad and $N v$-opa dynamics, while some changes to the late network can be simulated using only the changed slp and prd expression at the eve5 stage. Our method can provide no direct evidence that specific interactions in the Drosophila GRN are conserved in Nasonia, but this analysis does imply that if there are substantial topological changes to the Nasonia network, these do not result in changes to the patterning process.

Finally, the similarities between Nasonia and Drosophila segmentation, at the level of the 
GRN involved, implies that these derive from a common ancestor GRN that likely evolved deep in the insect lineage. This GRN has proven developmentally flexible over evolutionary time, allowing different forms of morphological segmentation to be built on an overall conservative network. The changes we have detected in the GRN that underlie Nasonia segmentation are limited implying that only minor modifications of an ancestral but flexible GRN may be enough to generate endless variety in morphological segmentation.

\section{Methods}

\subsection{Hybridization chain reaction}

Nasonia were raised on commercially sourced Sarcophaga bullata pupae (from Mantis Mayhem, https://mantismayhem.co.uk/shop/ols/products/green-blue-bottle-flies-pupae), at $25^{\circ} \mathrm{C}$. Nasonia cultures were a kind gift from Dr David Shuker and Dr Nicola Cook (St Andrews University).

Embryo collection was carried out after Werren and Loehlin, 2009. Flugs were modified such that $S$. bullata pupae could be placed, head-exposed, inside the plug. Embryos were collected from pupae provided to Nasonia for 12 hours, to provide the relevant stages of segmentation. Some embryos were stored in the fridge overnight for convenience. Hosts were cracked open under a dissecting microscope and dipped into $5 \mathrm{~mL}$ of heptane, $4.5 \mathrm{~mL}$ of $\mathrm{PBS}$ (phosphate buffered saline)) and $0.5 \mathrm{~mL} 37 \%$ formaldehyde in a $15 \mathrm{~mL}$ falcon, and fixed for 8-18 hours. After fixing, embryos collected at the bottom of the falcon. To dechorionate the embryos, the bottom formaldehyde layer was replaced with $100 \%$ ice cold methanol, and shaken vigorously for 1-3 minutes. Dechorionated and devitellenized embryos settled at the bottom of the falcon and were transferred to an eppendorf, washed $3 \mathrm{x}$ in methanol, and stored at $-20^{\circ} \mathrm{C}$.

Hybridization chain reaction was performed per Choi et al., 2016. Proteinase K digestion was not required for efficient probe penetration, so embryos were dehydrated in a methanol series before incubation in $100 \mu \mathrm{L}$ hybridization buffer for 30 minutes at $37^{\circ} \mathrm{C}$ was added. 1 
pmol probe (ie $1 \mu \mathrm{L}$ of $1 \mu \mathrm{M}$ probe) was added to $50 \mu \mathrm{L}$ hybridization buffer, and embryos were incubated with probe overnight at $37^{\circ} \mathrm{C}$. Embryos were then washed four times with probe wash buffer for 15 minutes each at $37^{\circ} \mathrm{C}$, before being washed three times for five minutes each in 5 x SSCT ( 5 x sodium citrate- Tween buffer). Embryos were pre-amplified in amplification buffer for 30 minutes. Hairpins were prepared by heating each hairpin individually to $95^{\circ} \mathrm{C}$ for 90 seconds, and leaving in a drawer at room temperature for 30 minutes. 3 pmol hairpin was added to $50 \mu \mathrm{L}$ of amplification buffer and added to the embryos. Embryos were amplified overnight in the dark at room temperature. Embryos were then washed $2 \mathrm{x} 5$ minutes, $2 \mathrm{x}$ 30 minutes, and 1x 5 minutes in SSCT. DAPI (Invitrogen) was added to the first 30 minute wash. Embryos were mounted in ProLong Glass (Invitrogen), left to cure at room temperature overnight, and stored in the fridge before imaging. Imaging was performed using a Olympus FV3000 confocal microscope at the Department of Zoology, Cambridge University, using the UPLSAPO30X 30X silicon oil lens.

\subsection{Modelling}

All scripts are available at https://github.com/Shannon-E-Taylor/masters.

Simulations of the pair rule system were performed using the modelling framework described in Clark, 2017, and the code from the supplemental information of that paper. The model consists of a one-dimensional row of cells. Boolean network analysis was performed using the BoolNet R ( $\mathrm{R}$ version 3.4.4) package (R Core Team, 2018; Müssel et al., 2010). The full GRN from the supplemental information of Clark, 2017 was used for modelling. To generate a state graph, the plotStateGraph function was used. To identify attractors, the getAttractors function was used; the default version of this code identifies all attractors for a synchronous network using the exhaustive method, which identifies trajectories for every possible initial condition. 


\subsection{Image analysis}

Image analysis was carried out using Fiji (Abràmoff et al., 2004; Schindelin et al., 2012). Analysis of blastoderm-stage embryos was done on partial or full maximum intensity projections; where embryos had more than one cell layer, relationships between genes were confirmed using the original 3D images. Background fluorescence was defined as the fluorescence visible in regions of the embryo clearly not expressing the gene of interest (for most embryos, the head) and was removed using the brightness-contrast tool in Fiji.

\subsection{Embryonic RNA interference}

Embryonic RNAi (eRNAi) was performed in Aotearoa New Zealand using a different strain of wasps to those in Cambridge. These Nasonia were reared on Lucilia sericata blowflies (www. biosuppliers.com), using similar methods as Werren and Loehlin, 2009 (S. bullata are not commercially available in Aotearoa).

dsRNA against egfp and $N v$-opa was ordered from Genolution (http://genolution.co.kr/agrorna/service-overview/).

To prepare embryos for microinjection, adult wasps were exposed to hosts for 2-5 hours. This long exposure time led to embryos of very similar stages, because the Nasonia took several hours to prepare to lay. Embryos were gently collected using fine forceps and aligned on a $1 \%$ agarose/PBS plate. Embryos were affixed to coverslips using heptane glue and covered with a small drop of Drosophila microinjection oil $(1.75 \mathrm{~mL}$ Halocarbon oil $700+$ $0.25 \mathrm{~mL}$ Halocarbon oil 27, Sigma). For the experiment reported here, embryos were then left in the fridge for four hours until it was time to inject them, these embryos were blastoderm stage embryos prior to cellularization. Other experiments, injecting embryos at the time of pole cell formation, resulted in similar phenotypes.

Embryos were injected using the PLI-100 (Harvard Apparatus) injection apparatus, and borosilicate needles. The coverslip was then transferred to the agarose plate to maintain humidity, and incubated at $28^{\circ} \mathrm{C}$ until all embryos had fully developed, 24-36 hours. Imaging 
was performed using DIC optics and an Olympus BX61 compound microscope.

\section{Acknowledgements}

\section{References}

Abràmoff, Michael D., Paulo J. Magalhães, and Sunanda J. Ram (2004). "Image processing with ImageJ". Biophotonics Int. 11.7, pp. 36-41. DOI: 10.1201/9781420005615.ax4.

Akam, Michael (1989). "Making stripes inelegantly". Nature 341, pp. 282-283. DoI: 10.1038/ 341282a0.

Auman, Tzach and Ariel D. Chipman (2018). "Growth zone segmentation in the milkweed bug Oncopeltus fasciatus sheds light on the evolution of insect segmentation". BMC Evol. Biol. 18, Article 178. DOI: 10.1186/s12862-018-1293-z.

Baudouin-Gonzalez, Luis et al. (2020). "The evolution of Sox gene repertoires and regulation of segmentation in arachnids". bioRxiv Prepr. DOI: 10.1101/2020.06.04.133389.

Binner, Priska and Klaus Sander (1997). "Pair-rule patterning in the honeybee Apis mellifera: Expression of even-skipped combines traits known from beetles and fruitfly". Dev. Genes Evol. 206, pp. 447-454. DOI: 10.1007/s004270050074.

Brent, Ava E et al. (2007). "Permissive and instructive anterior patterning rely on mRNA localization in the wasp embryo." Science (80-. ). 315, pp. 1841-3. DOI: 10.1126/science. 1137528.

Briscoe, James (2019). "Understanding Pattern Formation in Embryos: Experiment, Theory, and Simulation". J. Comput. Biol. 26.7, pp. 696-702. DOI: 10.1089/cmb.2019.0090. 
Buchta, Thomas et al. (2013). "Patterning the dorsal-ventral axis of the wasp Nasonia vitripennis". Dev. Biol. 381.1, pp. 189-202. DOI: 10.1016/j ·ydbio.2013.05.026.

Bull, Alice Louise (1982). "Stages of living embryos in the jewel wasp Mormoniella (Nasonia) Vitripennis (Walker) (Hymenoptera: Pteromalidae)". Int. J. Insect Morphol. Embryol. 11.1, pp. 1-23. DOI: 10.1016/0020-7322(82)90034-4.

Chipman, Ariel D. (2020). "The evolution of the gene regulatory networks patterning the Drosophila Blastoderm". In: Curr. Top. Dev. Biol. Academic Press Inc., pp. 297-324. DOI: $10.1016 /$ bs.ctdb.2020.02.004.

Choe, Chong Pyo and Susan J. Brown (2009). "Genetic regulation of engrailed and wingless in Tribolium segmentation and the evolution of pair-rule segmentation". Dev. Biol. 325, pp. 482-491. DOI: $10.1016 / \mathrm{j} \cdot$ ydbio.2008.10.037.

Choi, Harry M. T. et al. (2016). "Mapping a multiplexed zoo of mRNA expression." Development 143, pp. 3632-3637. DOI: 10.1242/dev.140137.

Choi, Harry M. T. et al. (2018). "Third-generation in situ hybridization chain reaction: Multiplexed, quantitative, sensitive, versatile, robust". Development 145.12, Article dev165753. DOI: $10.1242 / \mathrm{dev} .165753$.

Clark, Erik (2017). "Dynamic patterning by the Drosophila pair-rule network reconciles longgerm and short-germ segmentation". PLoS Biol. 15.9, Article e2002439. DOI: 10.1371/ journal.pbio. 2002439.

Clark, Erik and Michael Akam (2016). "Odd-paired controls frequency doubling in Drosophila segmentation by altering the pair-rule gene regulatory network". Elife 5, e18215. DOI: 10.7554/eLife.18215.

Clark, Erik and Andrew D. Peel (2018). "Evidence for the temporal regulation of insect segmentation by a conserved sequence of transcription factors". Development 145, Article dev155580. DOI: $10.1242 / \operatorname{dev} .155580$.

Clark, Erik, Andrew D Peel, and Michael Akam (2019). "Arthropod segmentation." Development 146, dev170480. DOI: 10.1242/dev. 170480.

Copf, Tijana, Reinhard Schröder, and Michalis Averof (2004). "Ancestral role of caudal genes in axis elongation and segmentation". Proc. Natl. Acad. Sci. 101.51, pp. 17711-17715. DOI: 10.1073/pnas. 0407327102 . 
Crombach, Anton et al. (2012). "Medium-throughput processing of whole mount in situ hybridisation experiments into gene expression domains". PLoS One 7.9. Ed. by Alistair P. McGregor, Article e46658. DOI: 10.1371/journal.pone.0046658.

Davis, Gregory K. and Nipam H. Patel (2002). "Short, Long, and Beyond: Molecular and Embryological Approaches to Insect Segmentation". Annu. Rev. Entomol. 47, pp. 669699. DOI: 10.1146/annurev.ento.47.091201.145251.

Dearden, Peter K. et al. (2006). "Patterns of conservation and change in honey bee developmental genes." Genome Res. 16.11, pp. 1376-84. DOI: 10.1101/gr.5108606.

DiFrisco, James and Johannes Jaeger (2019). "Beyond networks: mechanism and process in evo-devo". Biol. Philos. 34, Article 54. DOI: 10.1007/s10539-019-9716-9.

- (2021). "Homology of process: developmental dynamics in comparative biology".

Fleig, Richard and K. Sander (1988). "Honeybee morphogenesis: Embryonic cell movements that shape the larval body". Development 103, pp. 525-534.

Green, Jack and Michael Akam (2013). "Evolution of the pair rule gene network: Insights from a centipede". Dev. Biol. 382, pp. 235-245. DOI: 10.1016/j · ydbio.2013.06.017.

Jaeger, Johannes (2011). "The gap gene network". Cell. Mol. Life Sci. 68, pp. 243-274. DOI: $10.1007 / \mathrm{s} 00018-010-0536-\mathrm{y}$.

Janssen, Ralf and Graham E. Budd (2013). "Deciphering the onychophoran 'segmentation gene cascade': Gene expression reveals limited involvement of pair rule gene orthologs in segmentation, but a highly conserved segment polarity gene network". Dev. Biol. 382, pp. 224-234. DOI: 10.1016/j.ydbio.2013.07.010.

Janssen, Ralf et al. (2011). "Expression of myriapod pair rule gene orthologs". Evodevo 2, Article 5. DOI: 10.1186/2041-9139-2-5.

Janssen, Ralf et al. (2018). "Embryonic expression patterns and phylogenetic analysis of panarthropod sox genes: Insight into nervous system development, segmentation and gonadogenesis". BMC Evol. Biol. 18, Article 88. DOI: 10.1186/s12862-018-1196-z.

Jiménez, Alba et al. (2017). "A spectrum of modularity in multi-functional gene circuits". Mol. Syst. Biol. 13.4, Article 925. DOI: 10.15252/msb. 20167347. 
Keller, R G, C Desplan, and M I Rosenberg (2010). "Identification and characterization of Nasonia Pax genes". Insect Mol. Biol. 19, pp. 109-120. DOI: 10 . $1111 /$ j . 1365$2583.2009 .00921 . x$

Kimelman, David and Benjamin L. Martin (2012). "Anterior-posterior patterning in early development: Three strategies". Wiley Interdiscip. Rev. Dev. Biol. 1, pp. 253-266. DOI: 10.1002/wdev. 25 .

Koromila, Theodora et al. (2019). "Odd-paired is a late-acting pioneer factor coordinating with Zelda to broadly regulate gene expression in early embryos". bioRxiv Prepr. DOI: $10.1101 / 853028$.

Lim, Bomyi et al. (2018). "Temporal dynamics of pair-rule stripes in living Drosophila embryos". PNAS 115.33, pp. 8376-8381. DOI: 10.1073/pnas.1810430115.

Ma, Yue et al. (1998). "Gene regulatory functions of Drosophila Fish-hook, a high mobility group domain Sox protein”. Mech. Dev. 73, pp. 169-182. DoI: 10.1016/S0925-4773(98) 00050-1.

Müssel, Christoph, Martin Hopfensitz, and Hans A. Kestler (2010). "BoolNet-an R package for generation, reconstruction and analysis of Boolean networks". Bioinformatics 26.10, pp. 1378-1380. DOI: 10.1093/bioinformatics/btq124.

Nambu, Patricia A. and John R. Nambu (1996). "The Drosophila fish-hook gene encodes a HMG domain protein essential for segmentation and CNS development". Development 122.11, pp. $3467-3475$.

Novikova, Asya V. et al. (2020). "The multiple roles of caudal in early development of the milkweed bug Oncopeltus fasciatus". Dev. Biol. DOI: 10.1016/j.ydbio.2020.08.011.

Osborne, Peter W. and Peter K. Dearden (2005). "Expression of Pax group III genes in the honeybee (Apis mellifera)". Dev. Genes Evol. 215, pp. 499-508. DoI: 10.1007/s00427005-0008-9.

Paese, Christian L.B. et al. (2018). "A $S o x B$ gene acts as an anterior gap gene and regulates posterior segment addition in a spider". Elife 7, Article e37567. DOI: 10.7554/eLife . 37567. 
Panovska-Griffiths, Jasmina, Karen M. Page, and James Briscoe (2013). "A gene regulatory motif that generates oscillatory or multiway switch outputs". J. R. Soc. Interface 10, Article 20120826. DOI: 10.1098/rsif.2012.0826.

Peel, Andrew and Michael Akam (2003). "Evolution of segmentation: Rolling back the clock". Curr. Biol. 13, R708-R710. DOI: 10.1016/j.cub.2003.08.045.

Perez-Carrasco, Ruben et al. (2018). "Combining a Toggle Switch and a Repressilator within the AC-DC Circuit Generates Distinct Dynamical Behaviors". Cell Syst. 6, pp. 521-530. DOI: $10.1016 / j \cdot c e l s .2018 .02 .008$.

Peter, Isabelle S. and Eric H. Davidson (2016). "Implications of Developmental Gene Regulatory Networks Inside and Outside Developmental Biology". In: Curr. Top. Dev. Biol. Vol. 117. Academic Press Inc., pp. 237-251. DOI: 10.1016/bs.ctdb.2015.12.014.

Pultz, Mary Anne et al. (2005). "A major role for zygotic hunchback in patterning the Nasonia embryo". Development 132, pp. 3705-3715. DOI: 10.1242/dev.01939.

R Core Team (2018). R: A Language and Environment for Statistical Computing. R Foundation for Statistical Computing. Vienna, Austria.

Ray, Suparna et al. (2019). "The mlpt/ubr3/svb module comprises an ancient developmental switch for embryonic patterning". Elife 8, Article e39748. DOI: 10.7554/eLife. 39748.

Rosenberg, Miriam I et al. (2014). "Dual mode of embryonic development is highlighted by expression and function of Nasonia pair-rule genes." Elife 3, Article e01440. DOI: 10.7554/eLife.01440.

Russell, Steven R.H. et al. (1996). "The Dichaete gene of Drosophila melanogaster encodes a SOX-domain protein required for embryonic segmentation”. Development 122, pp. 36693676.

Schindelin, Johannes et al. (2012). "Fiji: An open-source platform for biological-image analysis". Nat. Methods 9.7, pp. 676-682. DOI: 10.1038/nmeth. 2019.

Schönauer, Anna et al. (2016). "The Wnt and Delta-Notch signalling pathways interact to direct pair-rule gene expression via caudal during segment addition in the spider Parasteatoda tepidariorum." Development 143, pp. 2455-63. DOI: 10.1242/dev.131656. 
Schroeder, Mark D., Christina Greer, and Ulrike Gaul (2011). "How to make stripes: Deciphering the transition from nonperiodic to periodic patterns in Drosophila segmentation". Development 138, pp. 3067-78. DOI: 10.1242/dev.062141.

El-Sherif, Ezzat et al. (2014). "Caudal Regulates the Spatiotemporal Dynamics of Pair-Rule Waves in Tribolium". PLoS Genet. 10, Article e1004677. DOI: 10.1371/journal.pgen . 1004677.

Soluri, Isabella V . et al. (2019). "Zygotic pioneer factor activity of Odd-paired/Zic is necessary for establishing the Drosophila Segmentation Network". bioRxiv Prepr. DOI: 10.1101/ 852707.

Vellutini, Bruno C. and Andreas Hejnol (2016). "Expression of segment polarity genes in brachiopods supports a non-segmental ancestral role of engrailed for bilaterians". Sci. Rep. 6, Article 32387. DOI: 10.1038/srep32387.

Verd, Berta, Nicholas A.M. Monk, and Johannes Jaeger (2019). "Modularity, criticality, and evolvability of a developmental gene regulatory network". Elife 8, Article e42832. DOI: $10.7554 /$ eLife. 42832 .

Verd, Berta et al. (2018). "A damped oscillator imposes temporal order on posterior gap gene expression in Drosophila". PLoS Biol. 16.2, Article e2003174. DOI: 10.1371/journal . pbio. 2003174.

Von Dassow, George and Eli Meir (2004). "Exploring Modularity with Dynamical Modules of Gene Networks". In: Modularity in Development and Evolution. Ed. by Gerhard Schlosser and Günter P. Wagner. Chap. 12.

Werren, John H and David W Loehlin (2009). "The parasitoid wasp Nasonia: An emerging model system with haploid male genetics". Cold Spring Harb. Protoc. 4.10. DOI: 10.1101/ pdb.emo134.

Wilson, Megan J. and Peter K. Dearden (2012). "Pair-rule gene orthologues have unexpected maternal roles in the honeybee (Apis mellifera)". PLoS One 7, Article e46490. DOI: 10 . 1371/journal. pone.0046490.

Wilson, Megan J., Melanie Havler, and Peter K. Dearden (2010). "Giant, Krüppel, and caudal act as gap genes with extensive roles in patterning the honeybee embryo". Dev. Biol. 339, pp. 200-211. DOI: 10.1016/j ·ydbio.2009.12.015. 
Wotton, Karl R et al. (2015). "High-resolution gene expression data from blastoderm embryos of the scuttle fly Megaselia abdita". Sci. Data 2, Article 150005. DOI: 10.1038/sdata. 2015.5.

Wouters, Arno G. (2003). "Four notions of biological function". Stud. Hist. Philos. Sci. 34.4, pp. 633-668. DOI: 3.

Xiang, Jie et al. (2017). "Conservation and variation in pair-rule gene expression and function in the intermediate-germ beetle Dermestes maculatus". Development 144, pp. 4625-4636. DOI: $10.1242 / \mathrm{dev} .154039$.

Zhu, Xin et al. (2017). "Speed regulation of genetic cascades allows for evolvability in the body plan specification of insects". Proc. Natl. Acad. Sci. 114.41, E8646-E8655. DOI: 10.1073/pnas. 1702478114 . 